\title{
A Poor Prognosis for the Diagnostic Screening Critique of Statistical
}

\section{Tests}

\section{Mayo ${ }^{1}$ and R D Morey ${ }^{2}$}

It is well known that in interpreting the results of a statistical test, one should not confuse $\operatorname{Pr}$ (test rejects $H_{0} \mid H_{0}$ is false) with $\operatorname{Pr}\left(H_{0}\right.$ is false|test rejects $\left.H_{0}\right)$. A popular criticism of significance tests assumes that the latter probability, often called the positive predictive value (PPV), is the appropriate measure of the warrant for " $H_{0}$ is false," understood as "there's a real effect" or "there's a genuine discrepancy from $H_{0}$. We argue that a high PPV for a hypothesis doesn't align with its being welltested, plausible, or warranted. This can be seen from the perspective of P-values, likelihood ratios or confidence intervals. The assumption that it is valid to marshal elements from significance tests in order to compute a PPV threatens to lead to further misunderstanding and misuse of this statistical methodology.

\section{Introduction and Overview}

As cases of high profile failures of replication in science mount, many fields find themselves in a state of introspection on statistical methodology. No method has received more attention than statistical significance testing, e.g., the American Statistical Association's (ASA) Statement on P-values (Wasserstein and Lazar 2017). In the felt urgency to proffer reforms to restore scientific credibility, there has been inadequate examination of equivocal terms being bandied about. Most practitioners are content to use an eclectic set of methods - all the more reason not to expect the same values from methods that measure different things. Yet one of the more popular accounts intended as a reform or a replacement for significance tests conflates concepts from Bayesian and frequentist statistics. We take no position on Bayesian-frequentist debates; all positions are hurt by the present lack of clarity on fundamental issues. We aim to restore clarity to a corner of the debate over significance testing.

\section{Overview}

There is some irony in the fact that failures of replication are often discovered by means of the very method often blamed: statistical significance tests.

${ }^{1}$ Department of Philosophy, Virginia Tech; email: mayod@vt.edu

${ }^{2}$ School of Psychology, Cardiff University; email: moreyr@cardiff.ac.uk 
For instance, several large-scale replication attempts of established psychological findings suggest that the psychological literature is less robust than previously believed. These conclusions are reached by applications of significance tests. How can a distrusted method be the key to unearthing problems? The answer is not hard to find: the problems are not significance tests themselves, but rather well-known abuses of tests: (1) hunting for statistical significance and selectively reporting cases that achieve a low significance level (also called cherry-picking, P-hacking, fishing for significance, and other things besides); and (2) taking a single statistically significant difference as indicating a genuine statistical effect, and - even worse as licensing a substantive research claim about causes. The first fallacy has garnered most of the attention, but the second is also pernicious, especially when based on artificial or fragile experiments. Yet some practitioners subscribe to a version of "null hypothesis significance testing" (NHST) that licenses the fallacies in (2). This runs afoul of both Fisherian and Neyman-Pearson (N-P) tests.

It is no surprise, then, that if a single significant result, with P-hacking to boot, is taken as grounds for a genuine research effect, that the error controls that these tests advertise disappear, as Ioannides (2005) and many other critics have argued. What is surprising is that some critics, including Ioannidis, have launched criticisms of tests based on an entirely different model, a cross between a Bayesian and frequentist account. The goal of Fisherian and N-P tests is to achieve error control without prior probabilities in scientific hypotheses, e.g., that the mass of the sun causes light to be deflected (as in General Relativity). Frequentist prior probabilities are rarely available in such cases. Instead, probability is used to control the probabilities of erroneous inferences from data-a test's error probabilities. In the Fisherian view of tests, there is just the null hypothesis $H_{0}$. Fisher reasons: If $H_{0}$ is false, then it's not an error to reject it. If $H_{0}$ is true, the probability of rejecting it is low, given by the significance level. Neyman-Pearson (NP) tests go further: they include the null and alternative hypotheses such that together they exhaust a space of possibilities. They can show, 
we shall reject $[H]$ when it is true not more, say, than once in a hundred times, and in addition we may have evidence that we shall reject $H$ sufficiently often when it is false. (Neyman and Pearson 1933, p. 291).

In N-P tests, the probability of erroneously rejecting $H_{0}$ (a Type I error) is fixed at a small number $\alpha$, and then the methodology seeks to maximize the probability the test rejects $H_{0}$ when it is false (its power).

The criticisms we wish to examine allege that these error probability controls are not sufficient. In particular, we are concerned with criticisms that replace the Type I error with a posterior probability of null hypotheses that are true, amongst those rejected. We are to imagine, at the outset, the proportion of true null hypotheses among the population of (no-effect) null claims that researchers will test - akin to the prevalence of a disease in a population, in diagnostic screening. We call this the diagnostic screening (DS) model of tests. Here are its elements:

Suppose you set out to test whether a new drug compound reduces blood pressure. There are three parts to the DS model: First, your null hypothesis - "this blood pressure drug has no effect" - is viewed as a selection, perhaps random, from a population of null hypotheses. Second, many or most of these are assumed to be true nulls (e.g., many drugs have no effect), so the prevalence of true nulls is, say, 0.2. Third, your results are dichotomized into "positive" or "negative", according to whether they reach statistical significance at a fixed level - usually .05 (or .025) with a "positive" finding construed as "a real effect" or "discovery". One then appeals to Bayes' theorem, with the "prevalence of true nulls" serving as the prior probability that your null is true. The criticism based on the DS model is this: the proportion of true nulls among those found statistically significant at level (say, .025 ) - a proportion we call the false finding rate $(F F R)^{1}$ - differs from and is greater than the .025 set by the test. That is,

$\operatorname{Pr}\left(\right.$ test rejects $\left.H_{0} \mid H_{0}\right)$ can be .025

${ }^{1}$ Colquhoun (2014) calls this the false discovery rate (FDR). However, this term was already used by Benjamini (1995) for a different concept that does not require prior probabilities. 
while

$$
\operatorname{Pr}\left(H_{0} \mid H_{0} \text { is rejected }\right)>.025
$$

if, for instance, it is assumed at least $50 \%$ of the null hypotheses are true. ${ }^{2}$

Is the criticism just a matter of transposing a probabilistic conditional and lamenting that they needn't be equal? The bottom line is, yes. But that doesn't suffice to dismiss the criticism. The critics argue that the quantity that really matters is the false finding rate (FFR) or its complement, the positive predictive value (PPV). If they are correct, then we ought to be using their measures rather than the error probabilities of significance tests. Ought we?

There are contexts in which the screening viewpoint is useful. Beyond diagnostic screening of disease, high-throughput testing of microarray data seeks to control the rates of genes worth following up. Nevertheless, we argue that the PPV does not quantify how well-tested, warranted, or plausible a given scientific hypothesis is (including ones about genetic associations where a DS model is apt). Employing the DS model has introduced confusion into the literature, by mixing up the probability of a Type I error (often called the "false positive rate") with the posterior probability given by the FFR: $\operatorname{Pr}\left(H_{0} \mid H_{0}\right.$ is rejected). Equivocation is being encouraged. Consider, for instance, that in frequentist tests, reducing the Type II error probability results in increasing the Type I error probability: that is, there is a trade-off. In the DS model, the trade-off disappears: reducing the Type II error rate also reduces the FFR, as we'll explain.

Ioannidis's (2005) argument and its variants have struck a nerve across scientific fields; his paper has been cited thousands of times in the decade since its publication. He and others who mount critiques of significance tests from the perspective of the DS model are earnestly trying to combat bad statistics. Ioannidis has gone to great lengths to raise the alarm against biased practices in medicine, and

\footnotetext{
${ }^{2}$ In the equations in this paragraph, we have used the conditional stroke "|". We do so for consistency with the notation used by proponents of the PPV, even though, from a frequentist perspective, the notation is not correct because it implies that $H_{0}$ is a random variable, which it is not. Likewise, Type I, Type II error rates, and power are not conditional probabilities.
} 
to quash abuses of significance tests that have been deplored for at least 60 years. Many of these critics even consider themselves "frequentists", and balk at constructing prior probabilities as degrees of belief (e.g., Colquhoun, 2014). We show, however, that the computations required by the DS model do not go through; furthermore, they fail to clinch the problem of reproducible effects. The DS model, we argue, sanctions misinterpretations of both significance tests and of what is required to warrant genuine reproducible effects. The crisis of replication is real, but we should be wary of reforms founded on distortions of methods. We, too, reject automatic and recipe-like uses of tests that sit at the base of significance test criticisms - including the DS model of tests - but the "reforms" based on DS arguments lead away from what is needed to restore scientific health to what ails statistical inference.

\section{The Diagnostic Screening (DS) Criticism}

The result on which the contemporary DS criticism is based has a long history; it may be traced back to the Bayesian arguments of Jeffreys (1939), Edwards, Lindman and Savage (1963), Gibbons and Pratt (1975), and Berger and Sellke (1987). Sometimes the screening analogy is explicit (Calquhoun, 2014), sometimes implicit (Ioannidis, 2005), and sometimes it is posed as 'a base-rate critique' without reference to diagnostic screening (Sellke, Bayarri and Berger, 2001; Cohen, 1994). Our points apply regardless of whether the DS analogy is explicitly invoked. To understand how its current metamorphosis really took off, we should examine Ioannidis's (2005) version of the argument.

Several methodologists have pointed out that the high rate of nonreplication (lack of confirmation) of research discoveries is a consequence of the convenient, yet ill-founded strategy of claiming conclusive research findings solely on the basis of a single study assessed by formal statistical significance, typically for a $p$-value less than 0.05 . Research is not most appropriately represented and summarized by $p$-values, but, unfortunately, there is a 
widespread notion that medical research articles should be interpreted based only on $p$-values. (Ioannidis, p. 0696)

Adopting this line of argument, critics say they can prove "that most claimed research findings are false"(Ioannidis, ibid).

\section{Some Well-Known Fallacies}

From the start, Fisher warned that to use p-values to legitimately indicate incompatibility (between data and a model), we need more than a single isolated low p-value: we must demonstrate an experimental phenomenon.

[W]e need, not an isolated record, but a reliable method of procedure. In relation to the test of significance, we may say that a phenomenon is experimentally demonstrable when we know how to conduct an experiment which will rarely fail to give us a statistically significant result. (Fisher 1947, p. 14)

This cuts against the common idea that non-significant results may be merely ignored or thrown away. For significance tests to be used as Fisher suggests, one would have to keep track of a set of tests, to check if one knew how to conduct an experiment that rarely fails to give a significant result. Until then, a small p-value is only a possible indication of "surprise" (1955). Even vouchsafing a genuine experimental effect, Fisher was clear, did not warrant a substantive causal interpretation.

[A]ccording to Fisher, rejecting the null hypothesis is not equivalent to accepting the efficacy of the cause in question. The latter...requires obtaining more significant results when the experiment, or an improvement of it, is repeated at other laboratories or under other conditions. (Gigerenzer et al. 1989, pp. 95-6)

Critics adopting the DS model of tests fret that scientists who publish on the basis of a single small p-value will make a fool of themselves too often (Colquhoun, 
2014). Fisher would say — and we concur - that they make fools of themselves each time they move from an isolated small p-value to announcing a discovery!

But that is not how the criticism is framed. A number of crucial issues emerge from examining why the DS criticism, at first glance, looks right. As we proceed, we will note several questions to which we return once all the pieces are in place.

The DS model and its use in criticizing significance tests

To avoid blurring concepts, we deliberately use screening terms to discuss the DS model. Security screening in airports offers a good example. Adequate screening would very rarely miss a dangerous item in a carry-on bag. It would also rarely trigger an alarm for no reason. Yet we know most of the alarms are false alarms, because dangerous items are, thankfully, very rare. In diagnostic language, the positive predictive value (PPV) - the probability that a triggered alarm is in fact, triggered by a dangerous item - is low. Let "+" indicate an alarm (e.g., a security alarm or a positive test for a disease) and let "D" indicate the event of interest (e.g., a truly dangerous item or a disease). To obtain the PPV, we apply ordinary Bayes' rule using the given relative frequencies (or prevalences).

$$
\begin{aligned}
& \text { PPV: } \operatorname{Pr}(\mathrm{D} \mid+)=\frac{\operatorname{Pr}(+\mid D) \operatorname{Pr}(D)}{[\operatorname{Pr}(+\mid D) \operatorname{Pr}(D)+\operatorname{Pr}(+\mid \sim D) \operatorname{Pr}(\sim D)]}=\frac{1}{(1+B)} \\
& \text { where } \mathrm{B}=\frac{\operatorname{Pr}(+\mid \sim D) \operatorname{Pr}(\sim D)}{\operatorname{Pr}(+\mid D) \operatorname{Pr}(D)}
\end{aligned}
$$

$\sim \mathrm{D}$ is the complement of D. The FFR is the complement probability to the PPV; that is, 1 - PPV.

The sensitivity is the probability that a randomly selected item (person or bag) with D will be identified as "positive":

SENS: $\operatorname{Pr}(+\mid D)$.

The specificity is the probability a randomly selected item without $\mathrm{D}(\sim \mathrm{D})$ will be found negative (-):

SPEC: $\operatorname{Pr}(-\mid \sim D)$; 
These give the probabilities for desired outputs: ringing the alarm given the bag is dangerous (SENS), and not ringing the alarm if the bag contains no dangerous items (SPEC).

To use the DS model to mount the criticism of significance tests, the "dangerous" bag from the luggage screening example is replaced by a "true effect": e.g., that an experimental manipulation actually has a nonzero effect. The term $\operatorname{Pr}(+\mid \sim D)-$ the false positive rate, the complement of the specificity - is equated to the Type I error probability of the significance test, and the sensitivity P(+|D) is replaced by the test's power (to be explained). Importantly, SENS and SPEC do not, by themselves, provide sufficient information to compute the PPV. We also require the prevalence of dangerous items in the population and, crucially, an assumption of random sampling. Only then can we equate the prevalence of the D with $\operatorname{Pr}(\mathrm{D})$. How do we get $\operatorname{Pr}(\mathrm{D})$ ?

Instead of populations of carry-on bags and luggage, imagine an urn of "null hypotheses", half of which have the property of "false": that is, they correspond to situations in which there is some true effect. We will draw a hypothesis from the urn at random, each with equal probability. In this situation, $\operatorname{Pr}(\mathrm{D})=.5$. Randomly selecting a hypothesis, we run a test and output "+" (declare it "significant") or "-" (declare it "non-significant"). Then we can define a PPV analogous to the one in the DS scenario. The PPV is intended to represent the probability that a selected null hypothesis is "false" conditional on a positive significance test ( + ).

The DS criticism is that the PPVs of tests are too low; equivalently, the FFRs are too high. There are two ways to obtain a low PPV: first, by assuming a low specificity (identified with $1-\alpha$, for $\alpha$ the significance level), and second, by assuming a low prior prevalence, $\operatorname{Pr}(\mathrm{D})$.

With $\operatorname{Pr}(D)=\operatorname{Pr}(\sim D)=.5$ :

$$
\mathrm{PPV}=\frac{1}{\left[1+\frac{\operatorname{Pr}(+\mid \sim D)}{\operatorname{Pr}(+\mid D)}\right]}
$$


All we need for the PPV to be greater than .5 is for $\mathrm{P}(+\mid \sim \mathrm{D})$ to be less than $\mathrm{P}(+\mid \mathrm{D})$. It suffices that the probability of incorrectly triggering the alarm is less than the probability of triggering it when it should be triggered. This gives a prevalence "boost", which is akin to the Bayesian criterion of evidence as raising the posterior above the prior. But a boost in PPV does not mean a high PPV; and the critics want it to be high.

It is well known in diagnostic testing that low prevalence is the most important factor in low PPV. Even if we assume a maximal sensitivity $\mathrm{P}(+\mid \mathrm{D})$ of 1 , all that is needed to bring the PPV below .5 is for the prevalence, $\operatorname{Pr}(\mathrm{D})$, to be small, e.g., $<\operatorname{Pr}(+\mid \sim D)$. Notice this does not make screening in the case of low prevalence uninformative! Much as an isolated significant result is merely an indication for further checking, sending positive (+) luggage for further scrutiny ensures that, taken together, false findings are rare. On the retest, they will usually discover someone simply forgot to discard their drink before the security screening. Interestingly, the rarity of dangerous bags - that is, the low prevalence of D'smeans we can be quite comforted in a negative result; in the presence of such high sensitivity, the probability a negative result is an error is low. Thus, bag screeners would typically prefer not to lower the sensitivity; they would rather control false positives by relying on the follow-up retests given to any "+" result.

Yet the criticism based on the DS model of tests assumes that the PPV should be at least close to .95 , so that the FFR matches the .05 significance level. Some say that if a test with $\alpha=.05$ leads to a PPV less than .95, then evidence against the null is "exaggerated". Why should measurements of very different things to be equal?

Even allowing $P(D)=.5-50 \%$ of nulls are assumed true - and taking $\operatorname{Pr}(+\mid \mathrm{D})$ to be .8 , a common requirement for power, the PPV .94! That is, if $\operatorname{Pr}(+\mid \sim D)=.05$, and $\operatorname{Pr}(+\mid D)=.8$, we get a high PPV of .94 .

However, although users of significance tests typically set a small, nominal level for a probability of a Type I error, such as $5 \%$, critics rightly argue that cherry picking, fishing for significance, P-hacking, multiple testing and the like result in a low specificity; that is, the actual probability of reporting a negative result even if the null hypothesis is true is low: a high rate of false positives. Ioannidis's criticism 
is based on assuming the probability of a Type I error is made quite a bit higher than the nominal level, due to cherry-picking, fishing and the like. He calls this bias.

The Table below excerpts some of the top (better) and bottom (worse) PPV entries from Ioannidis's table (Table 4, p. 0700), keeping the notation of diagnostic tests. Some of the PPVs, especially for exploratory research with lots of data dredging, are very low.

\begin{tabular}{|l|l|l|l|l|}
\hline $\mathrm{P}(+\mid \mathrm{D})$ & PREV of D & Bias & Practical Example & PPV \\
\hline .8 & $50 \%$ & 0.10 & Adequately powered RCT, little bias & .85 \\
\hline .95 & $67 \%$ & 0.30 & $\begin{array}{l}\text { Confirmatory meta-analysis of good-quality } \\
\text { RCTs }\end{array}$ & .85 \\
\hline .8 & $9 \%$ & .3 & $\begin{array}{l}\text { Adequately powered exploratory } \\
\text { epidemiological study }\end{array}$ & .20 \\
\hline .2 & $.1 \%$ & .8 & $\begin{array}{l}\text { Discovery-oriented exploratory research with } \\
\text { massive testing }\end{array}$ & .001 \\
\hline
\end{tabular}

For example, with 30 percent bias, the actual $\mathrm{P}(+\mid \sim \mathrm{D})=.05+(.95)(.3)=.33$.

A significance tester might claim that in every case with bias, the p-values reported are illicit. They would call for adjusting for such biasing selection effects, or at least making it clear they have occurred. Nevertheless, it's plausible that users of significance tests are rarely this virtuous. Moreover, even without explicit fishing, as Gelman and Loken (2014) observe, there can be so much flexibility with data as to have much the same effect. Simmons, Nelson, and Simonsohn (2012) call for a "21 word solution" whereby authors would declare if they fiddled with variables or selectively reported. It's not clear where Ioannidis's bias adjustments come from or how they relate to adjustments such as false discovery rates, Bonferroni, and many others. Goodman and Greenland (2007) argue that Ioannidis punishes a field like epidemiology twice: once by giving a high prevalence to "no effect" nulls, and a second time with a bias assignment. Ioannidis's approach lumps together all studies of a given class, some of which may be tainted by biasing effects and some not. That makes sense if the concern is simply the overall error rates, but might be unfair or overly generous to the particular study and hypothesis. 
Others who mount the DS criticism of significance tests allege that the problem holds even accepting the test's small $\alpha$ level, say .05, even with no bias. Their main gambit is to sufficiently lower the prevalence of $\mathrm{D}$ - which now stands for probability of a "true effect" - so that the PPV is low (e.g., Colquhoun 2014). Colquhoun's example retains $\mathrm{P}(+\mid \sim \mathrm{D})=.05, \mathrm{P}(+\mid \mathrm{D})=.8$, but shrinks the prevalence $\operatorname{Pr}(\mathrm{D})$ of true effects down to $10 \%$. That is, $90 \%$ of the nulls in your "research universe" are true. This yields a P PV of $64 \%$, which means that in only $64 \%$ of significant results are you detecting a true effect. The FFR, $\mathrm{P}(\sim \mathrm{D} \mid+)$ is .36 , much greater than $\mathrm{P}(+\mid \sim \mathrm{D})=.05$.

Several questions arise in appraising the DS criticism of significance tests:

1. Can $\operatorname{Pr}(+\mid \sim D)$ and $\operatorname{Pr}(+\mid D)$ be identified with the quantities from significance testing: the test's significance level and the power respectively?

2. Is the PPV computation relevant for evaluating tests? Does a high PPV for D indicate strong evidence for a particular hypothesis?

3. Can the prevalence term, $\operatorname{Pr}(D)$, be defined and obtained? A negative answer to any of these questions would threaten the validity of DS criticisms. We take them up in turn. The DS model criticism is deeply dependent on the notion of a test's power, yet power is one of the most misinterpreted notions in all of statistics (Mayo 1996, Mayo and Spanos 2006, 2011), including by DS critics.

\section{What are Statistical Significance tests?}

We set out tests in a manner that encompasses both simple Fisherian tests and Neyman-Pearson (N-P) tests because the DS model of tests is built on elements of both. We follow Neyman (1976) in using "significance tests" to cover N-P and Fisherian tests. None of the differences in philosophy or interpretation (between Fisherian and N-P tests) will alter our arguments. There are three basic components to a statistical test: (A) hypotheses, and a set of possible outcomes or data; (B) a measure of accordance or discordance, $\mathrm{d}(\boldsymbol{X})$, between hypotheses and data, and (C) an appraisal of a relevant sampling distribution associated with $d(\boldsymbol{X})$. 
(A) Hypotheses. One or more statistical hypotheses $H$, generally couched in terms of an unknown parameter, are claims about some aspect of the process that might have generated the data, given in a statistical model of aspects of that process. This model is often highly idealized and approximate.

(B) Distance function (test statistic) and its distribution. A function of the data $\mathrm{d}(\boldsymbol{X})$, referred to as the test statistic, reflects how well or poorly the observed data $\boldsymbol{X}_{0}$ accord with a test hypothesis $H_{0}$, which serves as a reference point. Typically, the larger the value of $d\left(x_{0}\right)$, the farther the data are from what is expected under the test hypothesis. The term test statistic is reserved for statistics whose distribution can be computed solely under the assumption of $H_{0}$, often called the "null" hypothesis, although it need not assert nullness.

Suppose we are interested in the mean $\mu$ of a Normal population, and we know that the standard deviation of the population is $\sigma=15$. (This would typically be estimated, but nothing in the logic changes, and it will be simpler to make out our points.) If we draw a sample of size $N=100$ the distribution of the test statistic $\bar{X}-$ its sampling distribution - is $\operatorname{Normal}\left(\mu, \frac{15}{\sqrt{100}}\right)$. Let our goal be to test the hypotheses:

$$
H_{0}: \mu \leq 100 \text { against } H_{1}: \mu>100 .
$$

The test is the same if we're testing $H_{0}: \mu=100$ against $H_{1}: \mu>100$. The test statistic would be:

$$
\mathrm{d}(\boldsymbol{x})=\sqrt{n}\left(\bar{X}-\mu_{0}\right) / \sigma
$$

where $\mu_{0}=100$. This gives $\mathrm{d}(\boldsymbol{x})=10(\bar{X}-100) / 15$. This test statistic captures the distance, in standard errors, between the observation $\bar{X}$ and the hypothesized null value $\mu_{0}$.

(C) Test Rule and its associated error probabilities. Neyman-Pearson (N-P) tests will generally predesignate a difference $\mathrm{d}_{\alpha}$ as the cut-off for rejecting $H_{0}$. The test rule is: if $\mathrm{d}(\boldsymbol{X})>\mathrm{d}_{\alpha}$, reject $H_{0}$. So we get:

$$
\text { Type } \text { I error probability }=\operatorname{Pr}\left(\mathrm{d}(\boldsymbol{X})>\mathrm{d}_{\alpha} ; H_{0}\right) \leq \alpha .
$$

where the semicolon in the probability statement should be read "computed under the assumption of'. The inequality is to express the fact that $\alpha$ is typically an upper 
bound on the Type I error probability. We will use the "reject" language for brevity, but the actual output can take different forms. For example,

"Declare a result significant at level .025 " iff $\mathrm{d}(\boldsymbol{X})>1.96$

For convenience, we round to 2.0, so we would declare significance at the .025 level iff $\bar{X}>2(1.5)=3$. This is the uniformly most powerful one-sided test. If we desire a two-sided test we can construct it out of two one-sided tests, and thus the rule will be $|\mathrm{d}(\boldsymbol{X})|>2$, except that the stated significance level will be doubled, i.e., .05 because an error can occur in either direction.

We can equivalently define the test in terms of the p-value as a test statistic. The $p$-value associated with $\mathrm{d}\left(\boldsymbol{x}_{0}\right)$ is the probability of a difference as large or larger than $\mathrm{d}\left(\boldsymbol{x}_{0}\right)$, under the assumption that $H_{0}$ is true:

$$
p\left(\boldsymbol{x}_{0}\right)=\operatorname{Pr}\left(\mathrm{d}(\mathbf{X}) \geq \mathrm{d}\left(\mathbf{x}_{0}\right) ; H_{0}\right)
$$

Since $\mathrm{d}\left(\mathrm{x}_{0}\right)$ is continuous, it does not matter if we use $>$ or $\geq$. Note that the event $\{\mathrm{d}>$ $\left.\mathrm{d}_{\alpha}\right\}$ is the same as $\{p<\alpha\}$.

The N-P test (1933) was deliberately constructed to encompass Fisher's simple significance test. With the single null of a simple significance test, one is merely assessing the "compatibility" between the data and $H_{0}$, taking into account the ordinary expected variability "due to chance" alone.

If the p-value is very small - say .01 - we have an indication of incompatibility with $H_{0}$. That is because if $H_{0}$ were true, we'd probably have observed a smaller difference than we did, that is $\operatorname{Pr}\left(\mathrm{d}(\boldsymbol{X})<\mathrm{d}(\boldsymbol{x}) ; H_{0}\right)$ is fairly high.

This is a mere indication; it does not, by itself warrant an inference of a genuine statistical effect.

Neyman and Pearson (1933) showed that without explicit consideration of a direction of an alternative, there was too much latitude in constructing tests. With an alternative defined, one may consider not only the Type I error, but the Type II error, which is failing to reject the null when it is false to some degree. Since the alternative hypothesis $H_{1}$ contains more than a single value of the parameter, the 
Type II error is actually a function $\beta\left(\mu_{1}\right)$ computed for various $\mu_{1}$ values in the alternative region. In our case, this consists of all $\mu>100$. The power of the test at $\mu_{1}$, $\operatorname{POW}\left(\mu_{1}\right)$, is the complement of the Type II error probability at $\mu_{1}$ :

$$
\operatorname{POW}\left(\mu_{1}\right)=\operatorname{Pr}\left(d(\boldsymbol{X})>\mathrm{d}_{\alpha} ; \mu=\mu_{1}\right) \text {. }
$$

It is common to speak of the "power of a test", but since the power varies with $\mu_{1}$, it is more correct to speak of the power function of a test. We might speak of a lowpower test as one whose power function is low for given, known, or interesting values of $\mu_{1}$, but this qualification must be noted. 

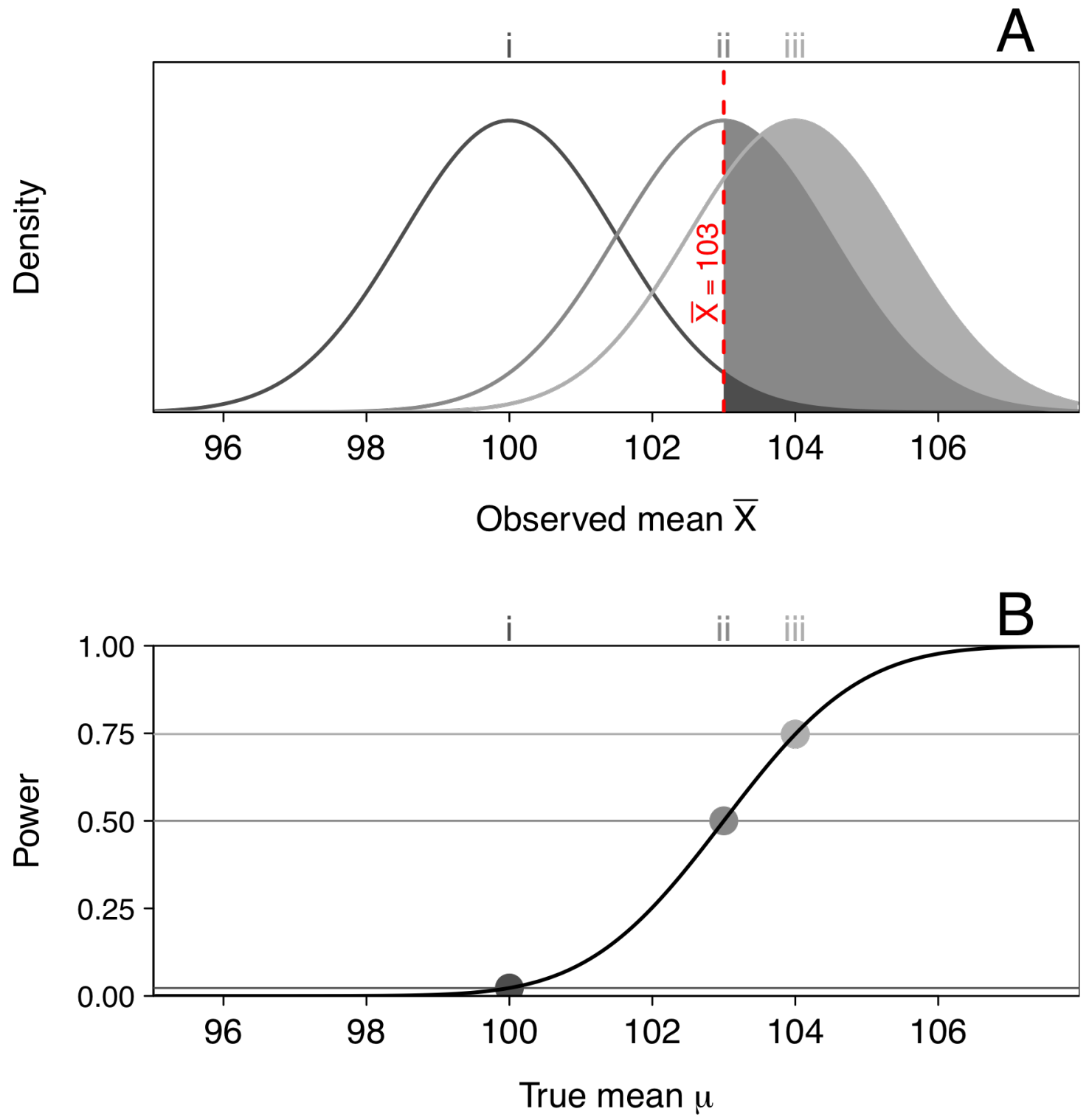

Figure 1: Sampling distributions for the observed mean $\bar{X}(A)$ and power curve for the true mean $\mu(B)$ for scenarios $i, i$, and, iii described in the text.

Three Important Things to Note About Power

To consider some values of the power function, compare POW(100), POW(103) and POW(104) in our test of $H_{0}: \mu=100$ against $H_{0}: \mu>100$, with the 
test statistic $\mathrm{d}(\boldsymbol{x})=10(\bar{X}-100) / 15$.

(i) $\operatorname{POW}(100)=\operatorname{Pr}(\bar{X}>103 ; \mu=100)=\operatorname{Pr}(Z \geq 2)=.025$.

$\mathrm{Z}=\sqrt{100}(\bar{X}-100) / 15$, a standard Normal variate.

(ii) $\operatorname{POW}(103)=\operatorname{Pr}(\bar{X}>103 ; \mu=103)=\operatorname{Pr}(Z \geq 0)=.5 .^{4}$

These two scenarios are depicted in Figure 1A and B. First, from (i) we see the power of the test, computed for the null hypothesis, is simply equal to $\alpha(.025)$. Under $H_{0}: \mu=100$, the probability is small that the result will give an indication of some discrepancy from the null, as weak as that claim is.

Second, the power for $\mu$ at the cut-off $d_{\alpha}=103$ is .5. Importantly, since we'd expect even larger differences half the time even due to chance variability, a test statistic near the cut-off is scarcely good evidence that $\mu>103$.

For a more extreme result, consider the power against $\mu=104$.

(iii) $\operatorname{POW}(104)=\operatorname{Pr}(\bar{X}>103 ; \mu=104)=\operatorname{Pr}(\mathrm{Z} \geq-2 / 3)=.75 .^{5}$

In our Normal example, the power only exceeds .5 for values of $\mu_{1}$ that exceed the cut-off $\mathrm{d}_{\alpha}$. We will use result (iii) in explicating a common fallacy of significance testing related to the DS model of tests.

${ }^{4}$ Here, $Z=\sqrt{100}(\bar{X}-103) / 15$, which is, again, a standard Normal variate.

${ }^{5}$ Here, $Z=\sqrt{100}(\bar{X}-104) / 15$. 
The "Mountains out of Molehills" Fallacy

It is sometimes thought that a statistically significant difference at a given level is a greater indication of a discrepancy from the null hypothesis if it came from a test with a higher, rather than a lower power. However, if we apply the reasoning of ordinary significance testing, we can see that this is in fact a fallacy. Mayo (1983, 1996, 2017) and Mayo and Spanos (2006) identify a rule of rejection RR:

$\mathrm{RR}: \mathrm{d}_{\alpha}$ is a poor indication that $\mu>\mu_{1}$ if there's a high probability that $\mathrm{d}>\mathrm{d}_{\alpha}$ even if $\mu=\mu_{1}$.

The result $\bar{X}=103$ is statistically significantly larger than $\mu=100$, and so it may be said to accord with alternative $H_{1}$ - that there is some positive discrepancy. But to infer $\mu>104$ would be unwarranted. If we took $\bar{X}=103$ to indicate $\mu>104$ we could be wrong $75 \%$ of the time. Were $\mu=104$, the probability of a larger difference than observed is .75. The inference to $\mu>104$, we may say, is poorly corroborated or inseverely tested by this result. 6 Put another way, in order for values $\mu>104$ to be outside the one-sided confidence interval around $\bar{X}=103$, the confidence coefficient would have to be no greater than a mere 25\% (see Figure 2). The test has high power to detect when $\mu>104$, but we would be foolish to take a significant result as evidence that $\mu>104$.

\footnotetext{
${ }^{6}$ The notion of severity can be made precise; see appendix A for an explanation.
} 


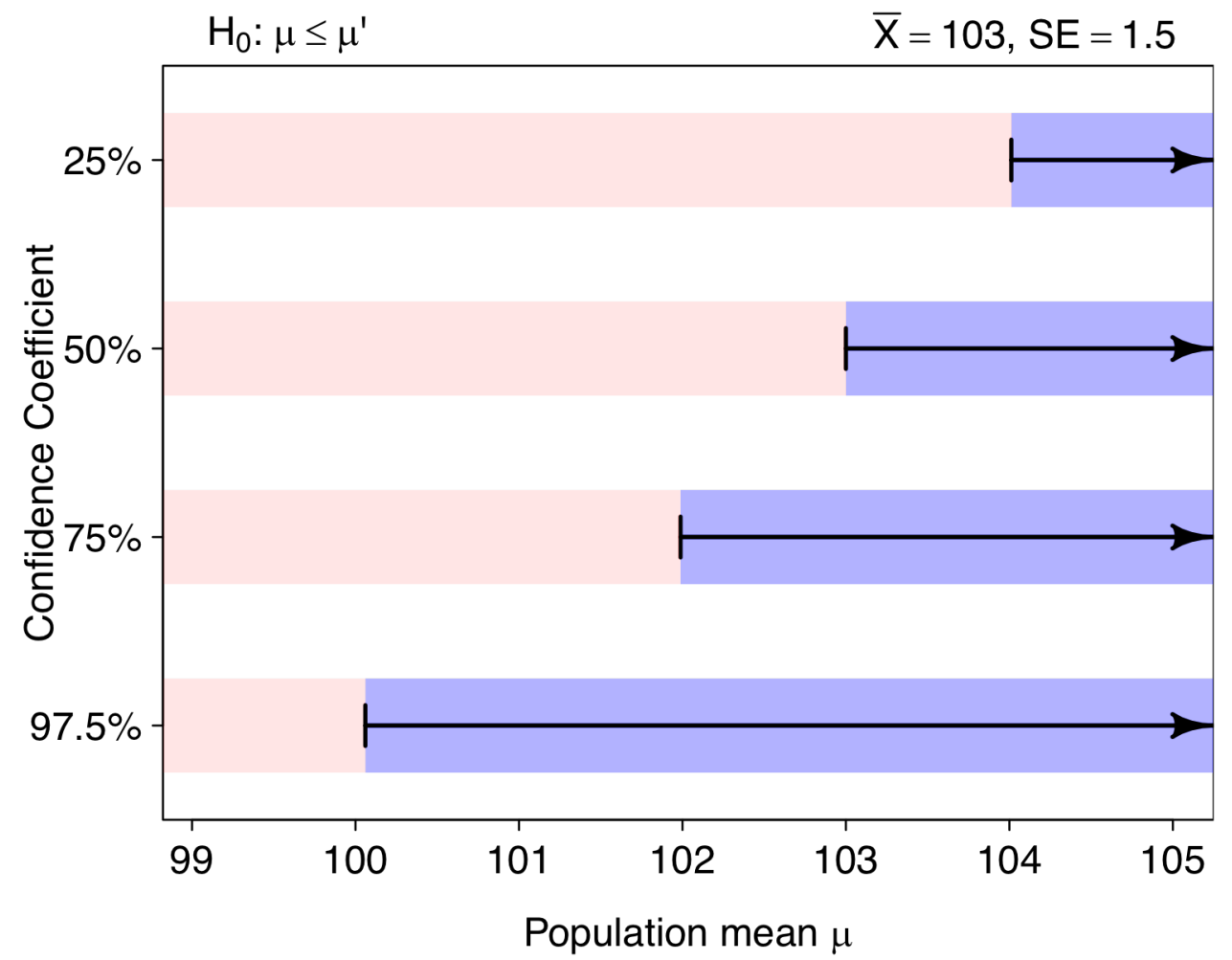

Figure 2: One-sided confidence intervals of various confidence coefficients when $\bar{X}=103$ and SE $=1.5$. If a value of $\mu^{\prime}$ is outside the confidence interval, then the hypothesis $\mu \leq \mu^{\prime}$ is rejected by the $\alpha=$ $1-C / 100$ level test, where $C$ is the confidence coefficient.

In order to focus on the logic of tests, we are putting to one side the important job of testing the assumptions of the model, without which the computations fail to go through.

\section{Significance Levels and Power Won't Give the PPV needed for the DS Criticism}

In the DS model of testing, the null and alternative are analogized to hypotheses whose probabilities sum to 1 ; that is, they exhaust the space of possible hypotheses (either luggage has a dangerous item in it or not). But in significance testing, the alternative is composite and the power is not a number, but a function. If we are testing exhaustive hypotheses, $H_{0}$ : there is a negative effect or no effect 
versus $H_{1}$ : there is some positive effect, then the power associated with $H_{1}$ won't be high. In fact, it will be very low, essentially equal to the significance level. The power computed at $H_{0}$ equals $\alpha$., and the power against slightly positive discrepancies are scarcely larger. Yet the criticism turns on an alternative with high power, say .8.

So consider an $H_{1}$ that our test has .8 power to detect. To get POW $\left(H_{1}\right)$ to be .8 requires setting the alternative $\sim .8$ standard errors above the cut-off $\mathrm{d}_{\alpha}$ : that is, $H_{1}: \mu>104.2(103+.8(1.5))$. But now the denial of the alternative $H_{1}$ does not yield the same null hypothesis used to obtain the Type I error probability of .05. Thus, the identification of "effect" and "no effect" with the hypotheses used to compute the Type I error probability and power are inconsistent with one another.

Non-Exhaustive Hypotheses Power cannot be used as shorthand for an underspecified "probability of obtaining a significant result assuming the null hypothesis is false and the effect is real." Yet the DS criticism assumes a single power (e.g., Ioannidis, 2005; Button et al. 2013). It is hard to see how this can work within the logic of significance tests. In a good N-P test the power increases as the hypothesized discrepancy increases-it's not one value.

The most plausible way to construe the DS argument is to assume the critics have in mind a test between a point null $H_{0}$, or a small interval around it, and a nonexhaustive alternative hypothesis $\mu=\mu_{1}$ against which there is a specified power like .8. It is known that there are intermediate values, but the inference will just compare two. With this setup, the PPV is again computed by means of an appeal to Bayes' theorem:

$$
\operatorname{Pr}\left(\mu=\mu_{1} \mid D\right)=\frac{\operatorname{Pr}\left(+\mid \mu=\mu_{1}\right) \operatorname{Pr}\left(\mu=\mu_{1}\right)}{\operatorname{Pr}\left(+\mid \mu=\mu_{1}\right) \operatorname{Pr}\left(\mu=\mu_{1}\right)+\operatorname{Pr}\left(+\mid \mu=\mu_{0}\right) \operatorname{Pr}\left(\mu=\mu_{0}\right)}
$$

$\mu_{1}$ is the effect size corresponding to "real effect." This posterior probability is entirely different from the $P P V$ described previously, however, because $\mu_{0}$ and $\mu_{1}$ do not correspond to $\sim \mathrm{D}$ and $\mathrm{D}$.

The DS model will give a high PPV to alternatives with high power, which is often taken to be .8 or .9 . For simplicity, take an alternative $\mu=\mu_{1}$ against which our 
test has power .84. All we do is add 1SE to the cut-off for rejection (although we are taking a value from the sample space and adding to one in the parameter space, there's no confusion here). In our example, $H^{\prime}: \mu=103+(1) 1.5=104.5$. Let $\operatorname{Pr}\left(\mu=\mu_{1}\right)=\operatorname{Pr}\left(\mu=\mu_{0}\right)=.5$, though the points we make will not depend on this assumption.

Now the DS computation gives the desired high PPV value:

$\operatorname{Pr}(\mu=104.5 \mid+)=.84 /(.84+.05)$, which is quite high. However, to take this high PPV as good evidence for $\mu \geq 104.5$ will immediately be seen to be fallacious (well, nearly immediately).

We know this from our discussion of the "mountains out of molehills" fallacy. Given that 103 is our cut-off and the power is .84 , we know that if $\mu$ were just below 104.5 - that is, the hypothesis is false - our test would yield a more statistically significant result $84 \%$ of the time. So this is very poor evidence that $\mu \geq 104.5$ ! Inferences from significance tests aren't in the form of points, but in the form of claims $\mu>\mu^{\prime}$ or $\mu<\mu^{\prime}$. Ironically, the DS model of tests appears to be licensing an inference $(\mu \geq 104.5)$ that exaggerates what a significance test would allow.

Moreover, by forcing the outputs into a crude dichotomy - for instance, 0 effect or $\mu \geq 104.5-$ PPV advocates are countenancing the crude dichotomy they decry. In drug testing, the type of example these critics often have in mind, the test is often designed to have high power to detect a clinically relevant effect. The clinically relevant discrepancy, as Stephen Senn suggests, is one "we should not like to miss", but importantly, obtaining a statistically significant result is not evidence we've found a discrepancy that big:

But where we are unsure whether a drug works or not, it would be ludicrous to maintain that it cannot have an effect which, while greater than nothing, is less than the clinically relevant difference. (Senn 2007, p. 201)

The problem can also be seen considering the likelihood ratio, defined as

$$
L R=\frac{f\left(X ; \mu=\mu_{1}\right)}{f(X ; \mu=100)},
$$




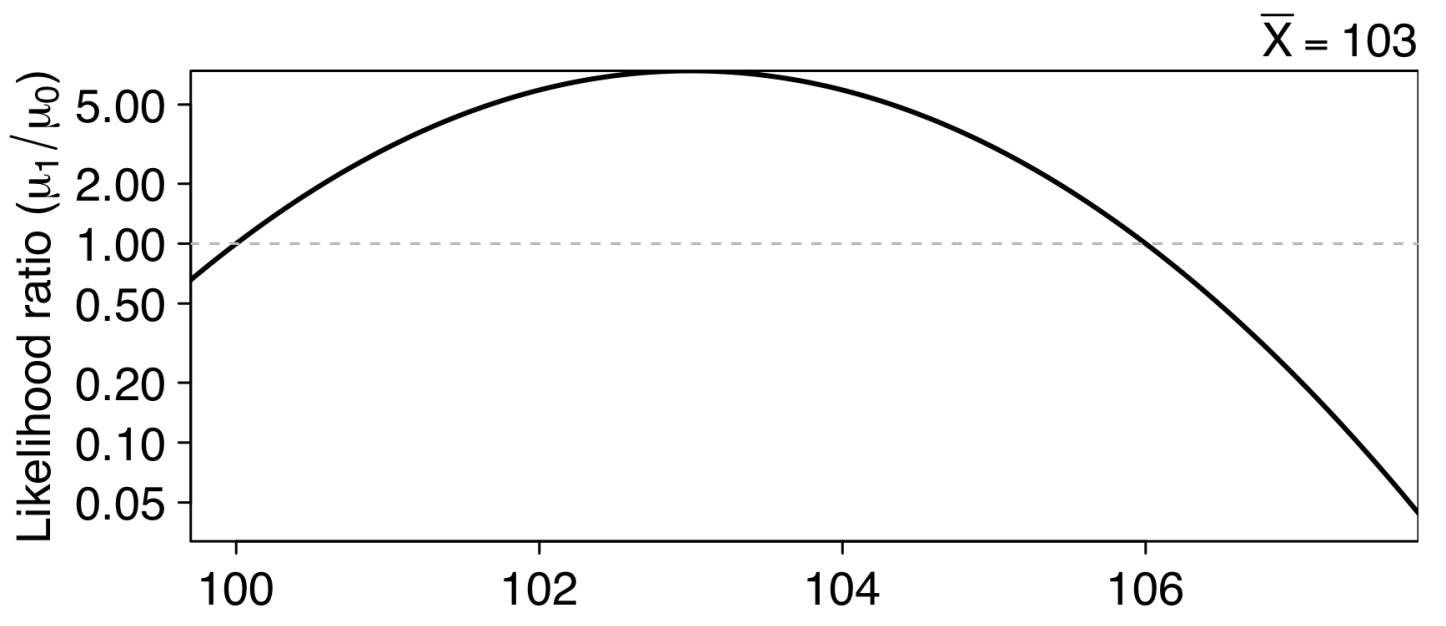

Alternative $\mu_{1}$

Figure 3: Likelihood ratio for various point alternative hypotheses against the null, assuming $\bar{X}=103$. Note that for larger values of the alternative, the likelihood ratio must increasingly favor the null hypothesis, because the observed data are much more concordant with the null than the unlikely alternative.

where $f$ is the probability density of the observed data. As $\mu_{1}$ becomes increasingly large, the just-significant observed result becomes comparatively more consistent with the null hypothesis, as shown in Figure 3. The PPV, on the other hand, will become increasingly large, favoring the effect, as the power to the alternative increases.

The root cause of the problem with PPV is that power and Type I error probabilities were never intended to be used in this manner. Exporting the power and Type I error rate from significance testing to serve as the terms in the DS model fails. What DS model advocates are doing — at least the simplest way to describe it - is that they are transposing the conditional in tests. This illicit transposition is, unfortunately, encouraged by the DS model. Consider Button et al, who say that

...the lower the power of a study, the lower the probability that an observed effect that passes the required threshold of claiming its discovery (that is, 
reaching nominal statistical significance, such as $p<0.05$ ) actually reflects a true effect. This probability is called the PPV of a claimed discovery. (Button et al, 2013, p. 366)

One shouldn't confuse a pre-data specification of a test, ideally with ample power, and the use of power, post-data, to assess the effect size indicated by a statistically significant result. We grant that there is often a legitimate reason to criticize a statistically significant result that emerged from a test with little power to detect effects of a size known to be expected. Why? Because it suggests it would be very difficult to have gotten the result legitimately. If it occurs in a field known to have considerable researcher flexibility, such a suspicion may well be warranted. The criticism is a roundabout way to say they think biasing selection effects occurred. Or it might be suspected that negative results are hidden away in file drawers. Again, this is to suggest the p-value is spurious or invalid. But that's very different from the argument employed by the advocates of the DS model of testing.

\section{What's the Reference Class for Prevalence?}

Someone might admit that using a test's error probabilities do not correspond to SENS and SPEC, yet aver there must be proportions out there corresponding to $\operatorname{Pr}(+\mid D)$ and $\operatorname{Pr}(-\mid \sim D)$, and that's all they need for the DS computation to go through. Can this work?

Consider the "prevalence" of "true" effects. What is it? Sellke, Bayarri \& Berger (2001) identify this probability - in a drug testing situation - with the proportion of tests that have an effective drug, saying that " $[t]$ he drugs can be for the same illness (say, AIDS, common cold, etc.) or different illnesses" (p. 63). Calquhoun (2014) identifies it with "the proportion of experiments we do over a lifetime in which there is a real effect." (p. 9). Ioannidis (2005) identifies it with "the number of 'true relationships'... among those tested in the field" (p. 0696). There are several distinct 
definitions of $\operatorname{Pr}(D)$ even in three papers that are ostensibly making the same argument. Every probability - including $\operatorname{Pr}(\mathrm{D})$, if it is a probability — must be defined relative to a reference class that answers the question "out of what population is the sample drawn"?

What's the relevant reference class for the prevalence $\operatorname{Pr}(\mathrm{D})$ ? A significance test used in research is not part of a well-defined sampling process. How does one count factors or hypotheses or relationships? Why not combine fields for estimating prevalence? Or why not make the reference class as specific as possible? Do we include all hypotheses that actually are tested, or ones that could be tested? Debates over how good the evidence is would be turned into disputes about which reference class one is entitled to use.

We scarcely have a list of all hypotheses that could possibly be tested in a field; much less do we know the proportion of these, or any subset we might choose, that are "true" in some sense. With continuous parameters, it could be claimed there are infinitely many hypotheses; individuating true ones could be done in multiple ways. Classifying by type of study might make sense, but any one umbrella will house studies from different fields with different proportions of true claims. For every relevant definition that would seem to make the PPV small, there would seem to be another equally defensible one that would make it large. Without a sampling process to decide the reference class, any numerical choice for $\operatorname{Pr}(\mathrm{D})$ is arbitrary. ${ }^{7}$

One might attempt a defense against our reference class critique by claiming that the PPV calculation it is not meant to be used in practice, but is merely a heuristic to show the difference between $\alpha$ and FFR, or between $(1-\alpha)$ and the PPV. It should always be kept in mind that even when a critic has claimed to perform a simulation, it is a simulation that assumes scarcely-questioned ingredients. If

\footnotetext{
${ }^{7}$ Interestingly, in his advocacy of the use of the PPV, Colquhoun (2014) warns us about the implications of a lack of a sampling scheme: "application of significance tests to observational data, e.g. epidemiological surveys of diet and health, is not valid" (pp.11-12). Exactly this critique can be made of modeling sequences of experiments as random, as DS model advocates do.
} 
aspects of the calculation fail, then of what value is the heuristic? Furthermore, it is clear that the PPV calculation is intended to assess the results of actual tests.

Even if we agreed on a reference class, say the proportion of true effects over your lifetime of testing, is $q$, this probability $q$ wouldn't be the probability that a selected effect is "true". It certainly would not be a frequentist probability for the randomly-selected hypothesis. We now turn to this.

\section{Probabilistic instantiation fallacy}

Colquhoun declares that

A Bayesian would refer to the prevalence as the prior probability that there is a real effect. There is no need to describe it in this way. It is a normal frequentist probability.... (Colquhoun, 2014, p. 10)

In fact, prevalence is not a Bayesian prior probability across possible null hypotheses; this would require the hypotheses to be exchangeable, which they are not. Nor is it a frequentist probability assignment for the hypothesis selected.

Suppose we did manage to do an experiment involving a random selection from an urn of null hypotheses, $100 q \%$ assumed to be true. The outcome may be $\boldsymbol{X}=$ 1 or 0 according to whether the hypothesis we've selected is true. Even allowing it's known that the probability of $\boldsymbol{X}=1$ is .5 , it does not follow that a specific hypothesis we might choose - say, your blood pressure drug is effective - has a probability of .5 of being true, for a frequentist (Mayo, 1997; 2005; 2010b). The fallacy is akin to assigning a particular .95 confidence interval estimate a probability of 95 .

Supposing that computing prevalence were possible, would it be relevant to the very thing that working scientists want to assess: strength of the evidence for effects or its degree of corroboration?

\section{High PPV due to High Prevalence Doesn't Capture Evidence}

It is supposed that in many fields of social and biological science that nearly everything is related to everything: that is, "all nulls are false". Meehl dubbed this 
background noise of small to medium relationships the "crud factor". Suppose one finds a single statistically significant result and announces a discovery, following the diagnostic screening model. If the "crud factor" is high, then there is a high prevalence of true claims (D's) among those found statistically significant. However, since the test they passed lacked stringency, one wouldn't be able to demonstrate a genuine replicable effect. Even finding a replicable experimental effect, but lacking an understanding of causes, and an inability to manipulate one factor to alter another, it would scarcely count as scientific knowledge. So a high PPV due to high prevalence doesn't indicate much about replicability.

By contrast, solid science may well be low prevalence. The search for "beyond the Standard Model" (BSM) in high energy particle physics, for example, has so far continually resulted in failing to reject the null. If and when they do find "bumps" that do not disappear on retesting, as in the case of the recent Higgs particle discovery, the weight given to the discovery is not measured in terms of a PPV, assuming we could attain it. If we have done quality science, know how to bring about results that rarely fail to be statistically significant- replicated the experiment, checked for flaws, tested for violated assumptions, and possess theoretical understanding, - then there's good warrant for taking the effect as real. It is important to understand that avoiding bias and achieving a severe test is what's doing the work, not prevalence.

\section{Conclusion}

We have argued that the analogy between diagnostic screening and significance tests on which the DS criticism relies is flawed. We've answered no to all of the questions we posed at the start. The quantities used to compute the PPV cannot be identified with terms involved in significance testing; a high PPV does not indicate strong evidence; and the crucial prevalence term cannot be defined.

We argued that the popularity of the DS model of tests has further confused the concepts of significance testing. Regardless of one's preferred statistical philosophy, this should be remedied. Notice what happens if we consider the "real Type I error" as $\operatorname{Pr}\left(H_{0} \mid+\right)$ (that is, the FFR). Since $\operatorname{Pr}\left(H_{0} \mid+\right)$ decreases with increasing 
power, it decreases with decreasing Type II error. So we know that to identify "Type I error" and $\operatorname{Pr}\left(H_{0} \mid+\right)$ is to use language in a completely different way than the one in which power is defined.

We showed that the DS model will give a high PPV to alternatives against which a test has high power, but the result, we have shown, is to greatly exaggerate the discrepancy for which there is evidence ("mountains out of molehills" fallacy). If the power to detect $\mu^{\prime}$ is high, then to move from a statistically significant result to inferring $\mu>\mu^{\prime}$ would have a high probability of being in error. Ironically, therefore, the DS model of tests licenses an inference $\mu>\mu^{\prime}$ that exaggerates what a significance test would allow.

We further argued that DS model blurs rates of false findings over an imagined population, PPVs, on the one hand, with an assessment of what has been shown about any particular effect, on the other. In other words, "positive predictive value," in this context, is a misnomer. The number isn't telling us how well-tested the hypothesized effect is. Holding it as the goal may well, inadvertently, enshrine trivial, safe science. Ioannidis says we should attempt to draw our hypotheses from urns with a high percentage of cases where there are real effects.

Large-scale evidence should be targeted for research questions where the prestudy probability is already considerably high, so that a significant research finding will lead to a post-test probability that would be considered quite definitive. (Ioannidis, 2005, p. 0700, emphasis ours)

If this means that scientists should choose to study hypotheses that have already passed severe tests, then the results could indeed be reliable (if not boosting our knowledge) but high PPV isn't what did the work.

Finally, we showed that there is no unique reference class for defining the prevalence probability $\operatorname{Pr}(\mathrm{D})$ because research is not a random process. The prevalence probability is not a Bayesian or frequentist probability, and even if it were, it would not be knowable.

The statistical inference patient was ailing before the popular DS model became common. We fear that the DS model is creating a further disability. 


\section{References}

Berger, J. O. \& Sellke, T. (1987). Testing a point null hypothesis: the irreconcilability of $\mathrm{p}$ values and evidence. Journal of the American Statistical Association, 82 (397), 112-122. Retrieved from http://www.jstor.org/stable/2289131

Button K., Ioannidis J., Mokrysz C., Nosek B., Flint J., Robinson E.et al. (2013). Power failure: why small sample size undermines the reliability of neuroscience. Nat Rev Neurosci 14: 365-376.

Casella, G., and Berger, R. (1987). 'Reconciling Bayesian and Frequentist Evidence in the One-sided Testing Problem', Journal of the American Statistical Association 82(397): 106-11.

Cohen, J. (1994). 'The Earth Is Round (p<. 05)', American Psychologist 49(12): 9971003.

Colquhoun, D. (2014). An investigation of the false discovery rate and the misinterpretation of p-values. Royal Society Open Science, 1 (3). Retrieved from http://rsos.royalsocietypublishing.org/content/1/3/140216

Cox, D. and Hinkley, D. 1974. Theoretical Statistics. London: Chapman and Hall.

Edwards, A., Lindman, H. and Savage, L. 1963. 'Bayesian Statistical Inference for Psychological Research', Psychological Review 70(3): 193-242.

Fisher, R. A. 1947. The Design of Experiments ( $4^{\text {th }}$ ed.). Edinburgh: Oliver and Boyd.

Gelman, A. and Loken, E. 2014. 'The statistical crisis in science', American Scientist 2: 460-65.

Gibbons, J. D., and J. W. Pratt. 1975. 'P-values: Interpretation and Methodology', The American Statistician 29(1): 20-5.

Gigerenzer, G., Swijtink, Porter, T. Daston, L. Beatty, J, and Kruger, L. 1989. The Empire of Chance. Cambridge: Cambridge University Press.

Goodman, S. and Greenland S. 2007. 'Why most published research findings are false: Problems in the analysis', PLoS Med. 4(4): 0773.

Ioannidis, J. P. A. (2005). Why most published research findings are false. PLoS Medicine, 2, 0696-0701.

Jeffreys, H. 1939. Theory of Probability. Oxford: Oxford University Press.

Lehmann, E. and Romano, J. 2005. Testing Statistical Hypotheses. $3^{\text {rd }}$ ed. Springer

Mayo, D. (1983). "An Objective Theory of Statistical Testing." Synthese 57(2): 297340.

Mayo, D. G. (1996). Error and the Growth of Experimental Knowledge. Science and Its Conceptual Foundation. Chicago: University of Chicago Press.

Mayo, D. G. (1997a). "Duhem's Problem, The Bayesian Way, and Error Statistics, or 'What's Belief got To Do With It?'" and "Response to Howson and Laudan," Philosophy of Science 64(1): 222-244 and 323-333.

Mayo, D. G. (1997b) "Error Statistics and Learning from Error: Making a Virtue of Necessity," in L. Darden (ed.) Supplemental Issue PSA 1996: Symposia Papers, Philosophy of Science 64: S195-S212.

Mayo, D.G. (2017), Statistical Inference as Severe Testing: How to get beyond the statistics wars Cambridge: Cambridge University Press. 
Mayo, D.G. and Cox, D. R. (2006) "Frequentists Statistics as a Theory of Inductive Inference," Optimality: The Second Erich L. Lehmann Symposium (ed. J. Rojo), Lecture Notes-Monograph series, Institute of Mathematical Statistics (IMS), Vol. 49: 77-97.

Mayo, D. G. and Spanos, A. (2011) "Error Statistics" in Philosophy of Statistics, Handbook of Philosophy of Science Volume 7 Philosophy of Statistics, (General editors: Dov M. Gabbay, Paul Thagard and John Woods; Volume eds. Prasanta S. Bandyopadhyay and Malcolm R. Forster.) Elsevier: 1-46.

Meehl, P. (1990). 'Why Summaries of Research on Psychological Theories Are Often Uninterpretable', Psychological Reports 66(1): 195-244.

Neyman, J. 1976. 'Tests of Statistical Hypotheses and Their Use in Studies of Natural Phenomena', Communications in Statistics-theory and Methods 5(8): 737-51.

Neyman, J. and Pearson, E. 1933. 'On the Problem of the Most Efficient Tests of Statistical Hypotheses', Philosophical Transactions of the Royal Society of London 231. Series A, Containing Papers of a Mathematical or Physical Character: 289-337.

Sellke, T., Bayarri, M. and Berger, J. O. (2001) Calibration of $\rho$ Values for Testing Precise Null Hypotheses, The American Statistician, 55(1): 62-71.

Senn, S. 2007. Statistical Issues in Drug Development, $2^{\text {nd }}$ ed. Chichster, New Sussex: Wiley Interscience, John Wiley \& Sons.

Simmons, J. Nelson, L. and Simonsohn, U. 2012. 'A 21 word solution', Dialogue: The Official Newsletter of the Society for Personality and Social Psychology, 26(2), 4-7.

Spanos, A. (2010) "Is Frequentist Testing Vulnerable to the Base-Rate Fallacy?" Philosophy of Science 77(4): 565-583.

Wasserstein, R. and Lazar, N. 2016. 'The ASA's Statement on P-values: Context, Process and Purpose', The American Statistician 70(2): 129-133. On-line commentary at: http://www.tandfonline.com/doi/pdf/10.1080/00031305.2016.1154108 


\section{Appendix on Severity}

A severity assessment provides a way to use a test's error probabilities to assess the well testedness of various statistical claims by given data. It's rationale is to provide a way to go beyond long-run behavioral uses of error probabilities while avoiding fallacies of rejection and non-rejection. It was first employed in Mayo 1988, 1991, 1996, 2003, and developed further in Mayo and Spanos 2006, 2011. It is based on a minimal requirement for evidence (as found in Popper, C.S. Peirce and others):

Data do not provide evidence for a claim $\mathrm{H}$ if a method had little or no capability of finding flaws in $\mathrm{H}$, even if they exist.

Put differently, data provide evidence for $\mathrm{H}$ only if produced by a method with reasonable chance of finding evidence against $\mathrm{H}$ if $\mathrm{H}$ is false.

Severity is a function of 3 arguments. Abbreviate the severity with which claim $C$ passes test $\mathrm{T}$ with data $\boldsymbol{x}$ :

SEV(test T, outcome $\boldsymbol{x}$, claim $C$ ).

If we know what test we're talking about, and what outcome, we can abbreviate with SEV(claim C). The most appropriate language to use to describe severe and insevere tests depends on the context; here we simply want to describe the concept. Pre-data, we need a good test, one with a sensible distance measure as a test statistic, satisfying requirements of increasing power with increasing discrepancies. Here we refer to a UMP test with a cutoff for rejection, say at the .025 level. The focus is just on the case of a result that rejects the null hypothesis in testing $H_{0}: \mu \leq 100$ against $H_{1}: \mu>100$.

In general, when we reject $\mathrm{H}_{0}$, we are interested in inferences of form $\mu>\mu_{1}$ where $\mu_{1}=100+\gamma$. We may call $\gamma$ a discrepancy parameter.

- Data do provide good evidence for $H: \mu>\mu_{1}$ to the extent that the test would probably have produced a smaller $\mathrm{d}$ than $\mathrm{d}_{0}$ if $\mathrm{H}$ is false and $\mu \leq \mu_{1}$.

- Data $\mathrm{d}_{0}$ provide poor evidence for $H: \mu>\mu_{1}$ if there's little or no chance of $\mathrm{d}<$ $\mathrm{d}_{0}$ even if $\mathrm{H}$ is false and $\mu \leq \mu_{1}$.

If the data reaches the cut-off for rejecting $\mathrm{H}_{0}$, we want to avoid claiming to have evidence for a magnitude of discrepancy beyond what is warranted; on the other hand, we don't 
want to merely report there's evidence for some discrepancy from $\mathrm{H}_{0}$, if more may be warranted. 1

I. Assessing severity for a result that just reaches .025 significance level (in testing $H_{0}: \mu \leq$ 100 against $\left.H_{1}: \mu>100\right)$.

The result $\bar{X}=103$ is statistically significantly larger than $\mu=100$, and so may be said to accord with alternative $H_{1}: \mu>100$. To assess severity we must consider the probability of getting a result that accords less well with $H_{1}$ were $H_{1}$ false.

1. Let $\gamma=1$. The severity with which $\mu>101$ passes our test with $\bar{X}=103$, that is, $\operatorname{SEV}(\mu$ $>101$ ), is the probability our tests would have yielded a smaller observed difference assuming $\mu=101$. The severity is $\operatorname{Pr}(\bar{X}<103 ; \mu=101)=\operatorname{Pr}(Z<1.33)$, which is .9. [Z = (103-101)/1.5]. This high severity is shown as point " $b$ " in Figure A1A. Thus, $\bar{X}=103$ is

Discrepancy $\gamma$

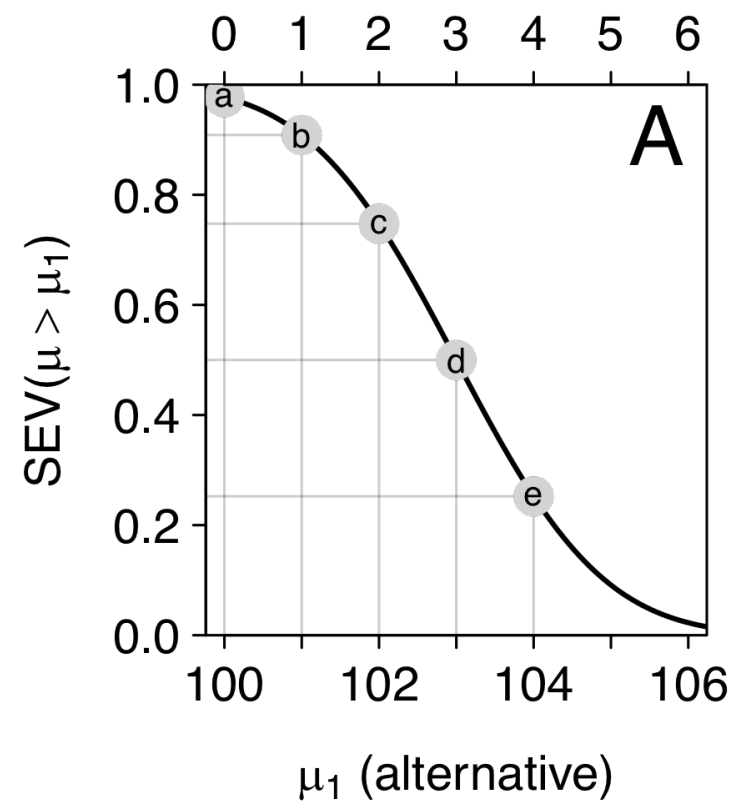

Discrepancy $\gamma$

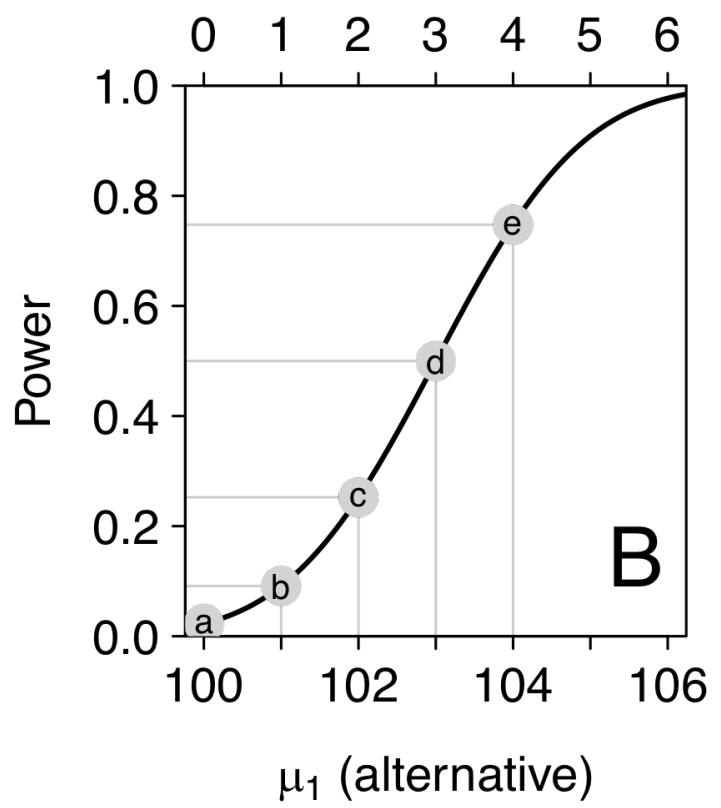

Figure A1. A: Severity curve for various alternatives of the form $\mu>\mu_{1}$. B: Power curve.

not only good evidence that $(\mu>100)$, but reasonably good evidence that $(\mu>101)$.

2. Let $\gamma=2$. The severity with which $\mu>102$ passes our test with $\bar{X}=103$ i.e., $\operatorname{SEV}(\mu>$ $102)=\operatorname{Pr}(\bar{X}<103 ; \mu=102)=\operatorname{Pr}(Z<2 / 3)=.75 .[Z=(103-102) / 1.5$.$] , as shown in Figure$ A1A, point "c". 
3. Let $\gamma=3$. $\operatorname{SEV}(\mu>103)$ with $\bar{X}=103=\operatorname{Pr}(\bar{X}<103 ; \mu=103)=\operatorname{Pr}(\mathrm{Z}<0)=.5$, as shown in Figure A1A, point "d".

The general assessment for a result just at the cut-off for rejection is: $\operatorname{SEV}\left(\mu>\mu_{1}\right)$ is 1 POW $\left(\mu_{1}\right)$. Since we have our power curve for this test, we know our severity curve is just the complement, shown in Figure A1B.

II. Assessing severity for a fixed inference, changing $\bar{X}$. Power is too restrictive because it is always defined in terms of a test's cut-off for rejection. If our statistically significant difference exceeds the cut-off for rejecting $\mathrm{H}_{0}$, we'd want to take that into account.

4. As shown above, with $\bar{X}=103$ there's poor severity for $(\mu>104)$. Suppose $\bar{X}=106$. Now what's $\operatorname{SEV}(\mu>104)$ ? $\operatorname{Pr}(\bar{X}<106 ; \mu=104)=\operatorname{Pr}(Z<1.33)=.9 .[Z=(106-104) / 1.5$.] So $\operatorname{SEV}(\mu>104)=.9$; see Figure A2, point " $c$ ".

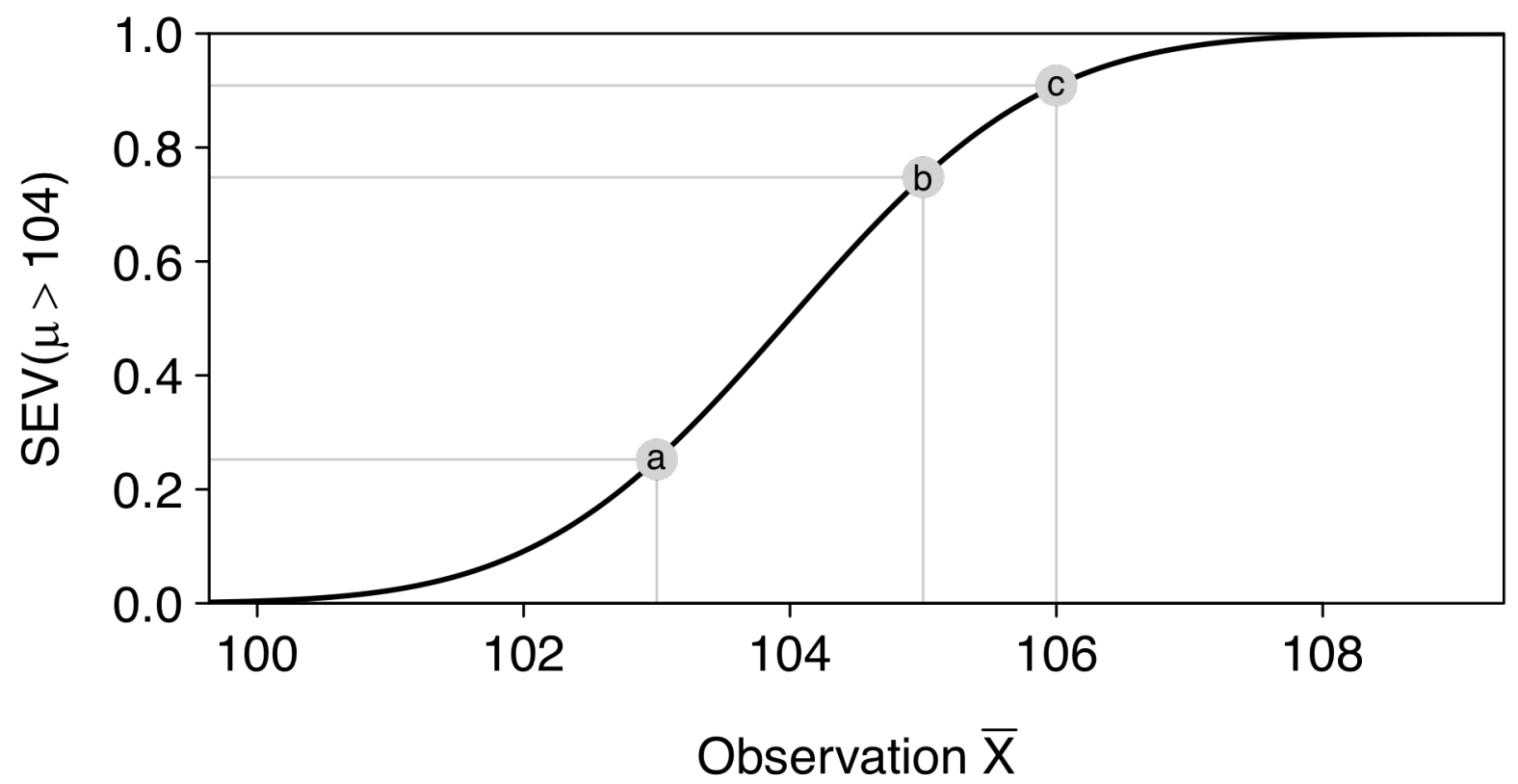

Figure A2: Severity assessments associated with the same inference $(\mu>104)$ but with different values of $\bar{X}$ within our rejection region. 
5. Say $\bar{X}=105$. Now what's $\operatorname{SEV}(\mu>104)$ ? $\operatorname{Pr}(\bar{X}<105 ; \mu=104)=\operatorname{Pr}(\mathrm{Z}<2 / 3)=.75$. $[\mathrm{Z}=$ (105-104)/1.5.] So $\operatorname{SEV}(\mu>104)$ with $\bar{X}=105$ is .75, as shown in Figure A2, point "b".

In the case of a non-significant result, keeping to the same test, the concern is to set an upper bound for the discrepancy. We know it's illicit to take a non-significant result to indicate the null is precisely true, because for a small enough discrepancy, our test would have very little probability to detect it even if present. For a discussion see Mayo and Spanos $(2006,2011)$.

${ }^{1}$ Until there has been a satisfactory check for biasing selection effects and model violations, the result is reported as "unaudited". 